\title{
Adesão à higienização das mãos como controle de infecção hospitalar na pandemia da COVID-19: Revisão bibliográfica
}

\author{
Adherence to hand hygiene as a control of hospital infection in the COVID-19 pandemic: \\ Literature review
}

Adherencia a la higiene de manos como control de la infección hospitalaria en la pandemia del COVID-19: Revisión de la literatura

Recebido: 03/07/2021 | Revisado: 10/07/2021 | Aceito: 14/07/2021 | Publicado: 24/07/2021

\author{
Maria Cyntia Kerle Calado Lima Malinconico \\ ORCID: https://orcid.org/0000-0002-6211-6418 \\ Hospital Pronto Socorro 28 de Agosto, Brasil \\ E-mail: cyntiakcl@ hotmail.com
}

\begin{abstract}
Resumo
Objetivo: Verificar a adesão à higienização das mãos por profissionais de saúde no combate à pandemia da COVID19. Metodologia: Foram revisados artigos encontrados nas bases de dados PubMed/MEDLINE, SCOPUS e Cochrane Library. Foram definidos os seguintes critérios de inclusão: estudos transversais realizados por meio de observação ou questionário que avaliaram a adesão dos profissionais de saúde à higienização das mãos durante a pandemia da COVID-19, publicados de janeiro de 2020 a maio de 2021. Após as buscas, os artigos selecionados pelos resumos foram lidos na íntegra. Foram excluídos os estudos que não especificaram o período em que foram realizados. Resultados: 331 artigos foram encontrados, no entanto somente 23 foram incluídos na revisão bibliográfica, mostrando que a observação mais prevalente foi em relação a exposição ou momento da higienização das mãos, avaliada em 11 estudos. Conclusão: A taxa de adesão à higienização das mãos na pandemia mostrou-se significantemente maior em relação ao período pré-COVID-19. Os médicos e enfermeiros são os profissionais que higienizam as mãos com maior frequência durante a pandemia, principalmente após o contato com pacientes. Já os técnicos e auxiliares de enfermagem se mostraram menos adeptos. A frequência de higienização foi maior nos picos de transmissão do SARS-CoV-2.
\end{abstract}

Palavras-chave: Controle de doenças transmissíveis; Controle de infecção; COVID-19; Higiene das mãos; Sanitização.

\begin{abstract}
Aim: To verify the adherence to hand hygiene by healthcare workers in the fight against the COVID-19 pandemic. Methodology: Articles found in PubMed/MEDLINE, SCOPUS and Cochrane Library databases were reviewed. The following inclusion criteria were defined: cross-sectional studies carried out through observation or questionnaire that evaluated the adherence of health professionals to hand hygiene during the COVID-19 pandemic, published from January 2020 to May 2021. After the searches, the articles selected by the abstracts were read in full. Studies that did not specify the period in which they were carried out were excluded. Results: 331 articles were found, however only 23 were included in the literature review, showing that the most prevalent observation was in relation to exposure or time of hand hygiene, evaluated in 11 studies. Conclusion: The rate of adherence to hand hygiene in the pandemic was significantly higher compared to the pre-COVID-19 period. Doctors and nurses are the professionals who most frequently clean their hands during the pandemic, especially after contact with patients. The technicians and nursing assistants, on the other hand, were less adept. The frequency of cleaning was higher in SARS-CoV-2 transmission peaks.
\end{abstract}

Keywords: COVID-19; Hand hygiene; Infection control; Sanitization; Transmissible disease control.

\section{Resumen}

Objetivo: Verificar la adherencia a la higiene de manos por parte de los profesionales de la salud en la lucha contra la pandemia COVID-19. Metodología: Se revisaron los artículos encontrados en las bases de datos PubMed / MEDLINE, SCOPUS y Cochrane Library. Se definieron los siguientes criterios de inclusión: estudios transversales realizados mediante observación o cuestionario que evaluaran la adherencia de los profesionales de la salud a la higiene de manos durante la pandemia COVID-19, publicados de enero de 2020 a mayo de 2021. Tras las búsquedas, los artículos seleccionados los resúmenes se leyeron en su totalidad. Se excluyeron los estudios que no especificaron el período en el que se realizaron. Resultados: Se encontraron 331 artículos, sin embargo solo 23 fueron incluidos en 
la revisión de la literatura, mostrando que la observación más prevalente fue en relación a la exposición o tiempo de higiene de manos, evaluada en 11 estudios. Conclusión: La tasa de adherencia a la higiene de manos en la pandemia fue significativamente mayor en comparación con el período anterior al COVID-19. Los médicos y enfermeras son los profesionales que se lavan las manos con mayor frecuencia durante la pandemia, especialmente después del contacto con pacientes. Los técnicos y auxiliares de enfermería, por otro lado, eran menos hábiles. La frecuencia de limpieza fue mayor en los picos de transmisión del SARS-CoV-2.

Palabras clave: Control de enfermedades transmisibles; Control de infección; COVID-19; Higiene de manos; Higienización.

\section{Introdução}

As infecções hospitalares ocorrem quando adquiridas após 72 horas da admissão do paciente no serviço hospitalar, prevalente em todo o mundo, tanto em países desenvolvidos quanto em desenvolvimento, e são relacionadas as principais causas de morte e ao aumento de morbidade em pacientes hospitalizados.

Um estudo feito em cinquenta e cinco países sob a coordenação da Organização Mundial da Saúde (OMS) revelou que em média 8,7\% dos pacientes tiveram algum tipo de infecção hospitalar (WHO, 2002). Estima-se que a cada 25 pacientes hospitalizados no Estados Unidos, 1 contraia infecção (Magill et al. 2014). A nível mundial essa média é alarmante, 1 a cada 10 pacientes hospitalizados adquire infecção (WHO, 2020). De acordo com o Ministério da Saúde (2019), a taxa de infecções hospitalares no Brasil é de $14 \%$.

Nesse contexto, a prevenção e controle das infecções hospitalares deve ser um dos objetivos centrais das políticas de saúde pública e privada, uma vez que a implementação de medidas de prevenção e a participação ativa dos profissionais de saúde estão relacionadas a diminuição de infecções em unidades de terapia intensiva (Marra et al. 2009; Marra, 2016) e em diferentes unidades hospitalares (Jarvis, 2007).

Dos diferentes meios de prevenção de infecção, a higiene das mãos é atualmente reconhecida como o pilar (Boyce \& Pitted, 2002a; Stella et al. 2019), evitando de forma efetiva que as mãos se tornem vetores de patógenos, consequentemente reduzindo ocorrência de infecções hospitalares (Pitted et al. 2006).

A frequência e a qualidade da higienização das mãos estão relacionadas ao menor risco de transmissão de doenças (Xun et al. 2021), reduzindo aproximadamente 30\% dos casos de doenças do trato gastrointestinal (Ejemot-Nwadiaro et al. 2015), 20\% das doenças respiratórias (Aiello et al. 2008), bem como 58\% de doenças infeciosas em pacientes com imunidade comprometida (Huang \& Zhou, 2007). Pesquisadores na Indonésia mostraram que a frequência e melhoria da lavagem das mãos diminuíram as taxas de infecção tifoide em cerca de 62\% (Alba et al. 2016). Outro estudo descobriu que lavar as mãos mais de quatro vezes por dia foi um fator protetor na redução do risco da influenza A, causada pelo vírus H1N1 (Chen et al. 2011).

A recomendação dos órgãos de controle de infecção é que a higiene das mãos seja feita antes e depois do contato com o paciente, quando as mãos estiverem visivelmente contaminadas, após o contato com objetos inanimados, incluindo equipamentos médicos, antes de inserir dispositivos invasivos que não requeiram um procedimento cirúrgico, antes de vestir luvas estéril, para inserção de um cateter e após a remoção das luvas (Boyce \& Pitted, 2002b; WHO, 2020). No entanto, os estudos mostram uma baixa adesão na frequência e na conformidade da lavagem das mãos pelos profissionais de saúde (Barnes et al. 2014), sendo observada frequência menor que 50\% (Samraj et al. 2008) e conformidade de $40 \%$ envolvendo todas as unidades do âmbito hospitalar. Segundo Erasmus et al. (2010), na unidade de terapia intensiva é ainda menor, de 30 $40 \%$.

Ahmed et al. (2020) realizaram um estudo entrevistando médicos, enfermeiras e técnicos de enfermagem, observando que eles apresentam conhecimento adequado sobre infecções hospitalares e sua relação com a conformidade da higiene das 
mãos. No entanto, os autores afirmam que há uma lacuna significativa entre o conhecimento e as práticas observadas (Ahmed et al. 2020), sofrendo influência por fatores individuais e do sistema (Pitted, 2000).

Apesar da higiene das mãos ser a principal arma na prevenção de doenças infecciosas dentro e fora dos hospitais, é um procedimento pouco discutido e estudado, possivelmente por ser considerada fácil e acessível, podendo ser realizada com sabonetes, degermantes e álcool gel (Boyce \& Pitted, 2002).

No último ano, a importância da sanitização das mãos ganhou um olhar diferente por todo o mundo, visto a ameaça epidemiológica do novo Coronavírus. No final de 2019, os primeiros casos, semelhantes à pneumonia, surgiram em Wuhan, China. No entanto, foi só no início do ano seguinte que as autoridades alertaram para um novo vírus da família Coronoviridae recém-descoberto, chamado de SARS-CoV-2 (Ortiz-Prado et al. 2020).

A COVID-19, doença respiratória causada pelo novo coronavírus tem sido responsável pela morte de milhões de pessoas em todo mundo, levando ao colapso sistemas de saúde de diversos países, incluindo os desenvolvidos, bem como o número de internações e o tempo de leito dos pacientes acometidos pelos quadros mais graves da doença. Esta situação veio reforçar ainda mais a importância da prática frequente das medidas de controle de infecção em ambientes hospitalares, a fim de evitar a contaminação dos profissionais de saúde envolvidos na linha de frente do combate à pandemia, de pacientes e acompanhantes (Wang, Pan \& Cheng, 2020).

A proliferação das infecções respiratórias ocorre principalmente devido ao contato com gotículas contendo vírus ou aspiração de aerossóis exalados de indivíduos infectados (Morawska \& Cao, 2020). No caso da Covid-19 a transmissão do vírus entre humanos ocorre por meio do contato com a secreção de gotículas respiratórias de um indivíduo infectado. As gotículas representam partículas de fluidos carregadas de vírus que são formadas no trato respiratório de uma pessoa infectada e expelidas pela boca e nariz durante a respiração, fala, tosse e espirros, representando a via direta de transmissão (Prather, Wang \& Schooley, 2020). Nesse contexto, a OMS declarou nos primeiros meses de 2020 que a higienização das mãos é um dos principais meios de prevenir a infecção pelo SARS-CoV-2 (WHO, 2020).

A higienização das mãos é essencial, pois há a possibilidade de contaminação pelo contato direto com as gotículas respiratórias, por tosses e espirros ou pelo contato indireto (Seto et al. 2003). Como discutido, os estudos anteriores à pandemia mostram pouca adesão pelos profissionais de saúde à higienização das mãos (Pitted, 2000; Samraj et al. 2008; Erasmus et al. 2010), justificado por problemas como irritação da pele, suprimentos inacessíveis, esquecimento, tempo insuficiente, falta de modelos positivos e a percepção da falta de evidências. Já a autoproteção tem sido identificada como o principal fator motivador associado à adesão por esses profissionais (Pitted, Mourouga \& Perneger, 1999; Boyce \& Pitted, 2002; Erasmus et al. 2009). Assim, ainda não se sabe como os profissionais de saúde aderiram a higienização das mãos durante a crise sanitária imposta pela pandemia. Este estudo objetivou verificar a adesão à higienização das mãos por profissionais de saúde no combate à pandemia da COVID-19.

\section{Metodologia}

Revisão bibliográfica do tipo narrativa, com abordagem qualitativa (Melis et al. 2019; Aidos et al. 2018). Foi realizado um levantamento bibliográfico para identificar os principais artigos que buscaram explicar a adesão à higienização das mãos pelos profissionais de saúdes em hospitais na pandemia da COVID-19. Foram revisados artigos encontrados nas bases de dados PubMed/MEDLINE, SCOPUS e Cochrane Library utilizando as seguintes palavras chaves: "infection control", "hand hygiene", "handwashing", "hand sanitation", "covid-19", "sars-cov-2", “coronavirus". O termo "AND” foi utilizado para agrupar os assuntos. Foram definidos os seguintes critérios de inclusão: estudos transversais realizados por meio de observação ou questionário que buscaram avaliar a adesão dos profissionais de saúde à higienização das mãos durante a pandemia da COVID-19 com resumo em inglês. Na primeira busca foram incluídos artigos publicados de janeiro de 2020 a 
maio de 2021 que abordavam o controle de infecção e higienização das mãos. Na segunda etapa os artigos selecionados pelos resumos foram lidos na íntegra a fim de verificar quais tinham avaliado a adesão à higienização das mãos durante pandemia. Foram excluídos os estudos que não especificaram o período em que foram realizados. Como suporte metodológico para definir os critérios de busca, inclusão e exclusão dos estudos foram empregados os estudos de Pereira et al. (2018), Ludke e Andre (2013) e Koche (2011). A revisão narrativa foi o modelo escolhido porque os dados são heterogêneos e não propícios a meta-análise. Por conveniência, os resultados das buscas foram discutidos e apresentados em tabelas.

\section{Revisão de literatura}

As pesquisas nas bases de dados localizaram 331 títulos e resumos, incluindo 199 no PubMed / MEDLINE, 196 no SCOPUS e 6 na Cochrane Library. Após a retirada das duplicatas, restaram 320 artigos, desses 45 foram selecionados para leitura do texto completo e 23 artigos foram incluídos na revisão bibliográfica (Figura 1).

Figura 1. Fluxograma dos estudos incluídos na revisão bibliográfica.

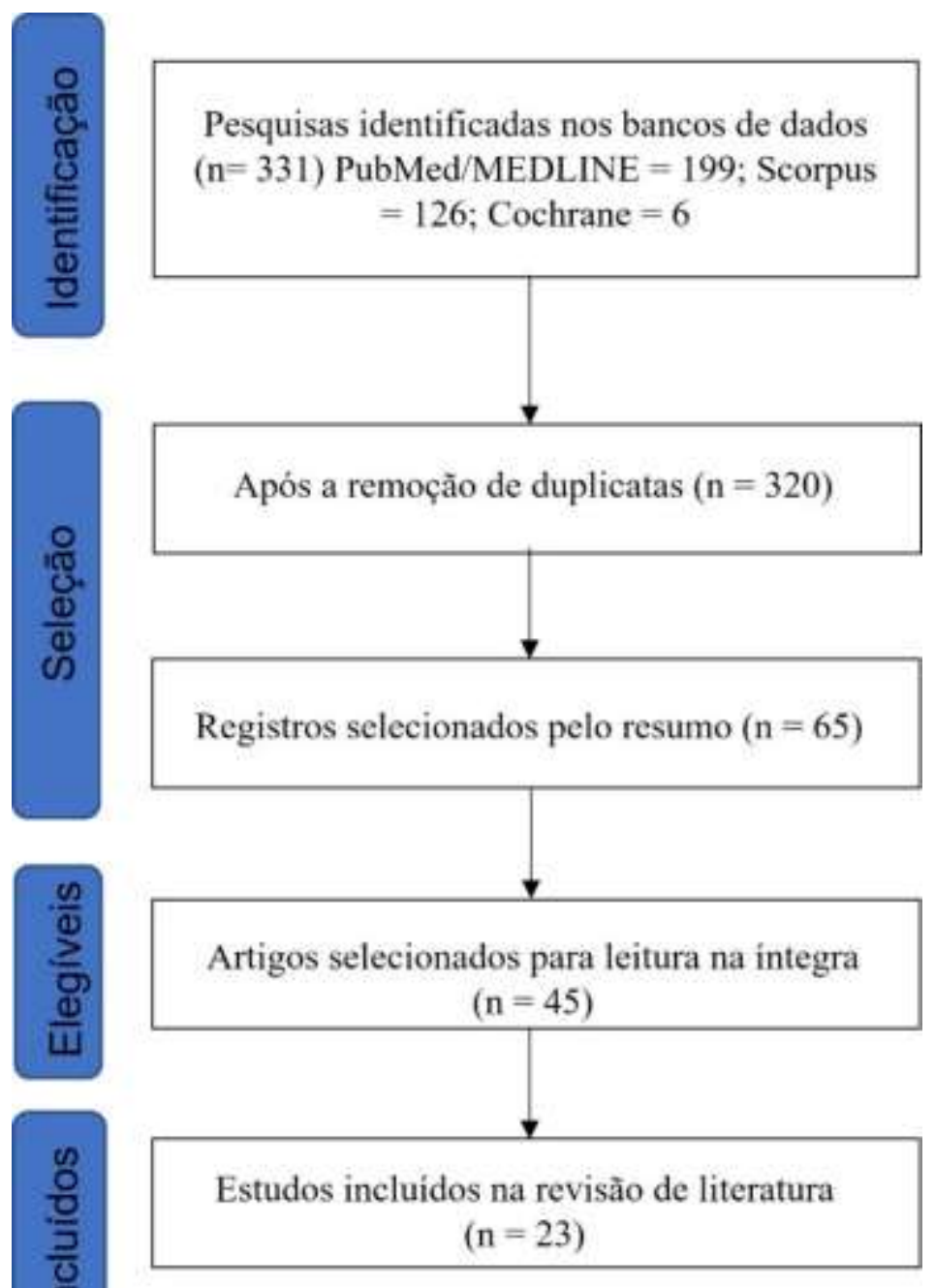

Fonte: Autores. 


\section{Resultados e Discussão}

Todos os estudos analisaram a adesão dos profissionais de saúde à higienização das mãos durante a pandemia da COVID-19. Os resultados foram divididos em tópicos para compor a discussão. Alguns estudos abordaram todos os tópicos em suas análises, dessa forma, a observação mais prevalente foi em relação a exposição ou momento da higienização das mãos, avaliada em 11 estudos. Para a adesão dos grupos profissionais foram encontrados 10 estudos e outros 6 estudos avaliaram a adesão em relação aos períodos da pandemia. A tabela 1 mostra a taxa de adesão à higienização das mãos nos 23 estudos incluídos nesta revisão.

\section{Profissionais de saúde}

Estudos anteriores relatam descumprimento das normas padrão de controle de infecção hospitalar por profissionais de saúde, relacionado a pouco conhecimento das práticas, excesso de sobrecarga de trabalho, falta de treinamento, ambiente de trabalho precário e falta de recursos (Efstathiou et al. 2011; Fayaz et al. 2014). Além disso os fatores de risco sociodemográficos como idade, sexo, estado civil, categoria de trabalho e experiência na área de atuação também estão associados a baixa adesão aos procedimentos de controle de infecção (Felix et al. 2013). Nesta revisão, os estudos encontrados mostraram a adesão e conformidade das práticas de controle de infecção pelos diferentes grupos de profissionais do âmbito hospitalar no período pandemia causada pelo SARS-CoV-2.

Argawal et al. (2021) avaliando 956 profissionais indianos entre julho e agosto de 2020, observaram que médicos residentes, técnicos e auxiliares de enfermagem apresentaram menor adesão às práticas preventivas de infecção comparados a médicos especialistas e enfermeiros $(\mathrm{p}<0,001)$. Os autores forneceram questionário aos profissionais com score de 1-5 para avaliar a conformidade da higienização das mãos, sendo escore 1, baixa conformidade e escore 5, alta conformidade. Os profissionais relataram seguir corretamente os passos de higienização das mãos (52,82\%) por pelo menos 20 segundos $(53,14 \%)$ em conformidade 5. O estudo de Ashinyo et al. (2021) avaliando 328 profissionais de 4 unidades hospitalares, referência para COVID-19 em Gana nos meses maio a agosto de 2020, também mostrou conformidade significantemente baixa na higienização das mãos para zeladoras $(78,4 \%)$, parteiras $(79,2 \%)$ e farmacêuticos $(66,7 \%)$ comparados a enfermeiros $(91,1 \%)$ e médicos $(85,5 \%)$. A maioria dos profissionais $(88,4 \%)$ relatou realizar a higienização das mãos sempre que recomendado ou a maioria das vezes. Da mesma forma, Elhadi et al. (2020) avaliaram 1572 médicos e enfermeiros de 21 hospitais da Líbia entre janeiro e março de 2020, onde observaram que os médicos faziam a higienização das mãos com maior frequência (44.2\%) quando comparados aos enfermeiros (33,3\%). A média de adesão da lavagem das mãos neste estudo ficou em 43,2\%. Em outro estudo realizado na primeira onda da pandemia na Europa, de janeiro a abril com 110 médicos do Reino Unido, 51\% confirmaram lavar as mãos frequentemente por 10-20 segundos (Hussain et al. 2021).

O estudo de Abbas et al. (2020) analisando 401 profissionais de saúde no Paquistão, observou que o número de médicos que higienizavam as mãos mais de cinco vezes ao dia por pelo menos 20 segundos foi significantemente maior comparando com médicos recém-formados e enfermeiros. Arriba-Fernandez, Molina-Cabrillana e Majem et al. (2021) mostraram resultados semelhantes, comprovando que os médicos realizam a higienização das mãos com maior frequência em todos os departamentos do hospital em relação a enfermeiros e técnicos. Avaliando os efeitos psicológicos da pandemia em mais de 200 médicos, Karakose e Malkoc (2021) mostraram que mais de 70\% tinham mais cuidado com a higienização das mãos durante a pandemia. Uma taxa de quase $90 \%$ de adesão à higienização das mãos foi observada no estudo de Egbi, Duru e Kasia et al. (2020), avaliando profissionais de um hospital universitário na Nigéria, referência para o tratamento da COVID-19, constatou que médicos, enfermeiros e técnicos não apresentavam diferenças na adesão à higienização das mãos. No entanto, em relação as práticas de prevenção de infecção, os profissionais que tinham pós-graduação tiveram melhor adesão. 
Tabela 1. Estudos que avaliaram a adesão à higienização das mãos pelos profissionais de saúde em hospitais na pandemia da COVID-19.

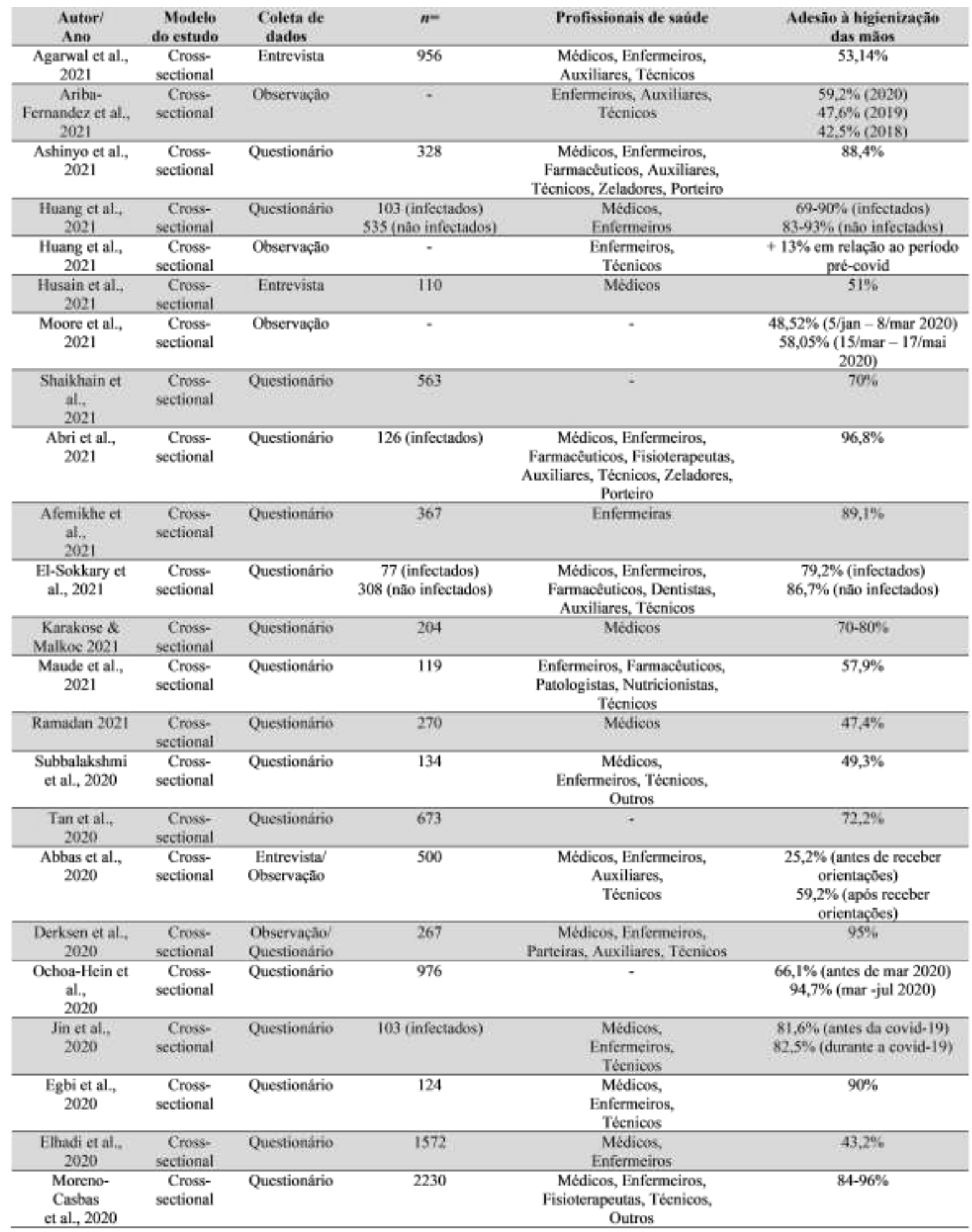

Fonte: Autores.

Contrariando os resultados anteriores que confirmam os médicos como os profissionais que apresentam melhor adesão à higienização das mãos durante a pandemia, Derksen, Keller e Lippke (2020) mostraram que 36\% das enfermeiras da 
ala de obstetrícia tinham maior cuidado com a higienização das mãos quando comparadas a parteiras (27\%) e médicos (24\%). Subbalakshmi et al. (2020) também mostraram que enfermeiros higienizavam as mãos sempre (65,8\%), enquanto médicos fizeram o procedimento quase sempre (53,3\%). Além disso, os enfermeiros mostraram ter melhor conhecimento sobre a técnica de lavagem das mãos preconizada pela OMS (78,9\%) quando comparados aos médicos $(41,3 \%)$. Finalmente, um estudo avaliou 367 enfermeiras de 6 hospitais linha de frente no combate à pandemia pelo SARS-CoV-2 na Nigéria, dessas, 327 (89,1\%) relataram seguir estritamente as práticas de higienização das mãos (Afemikhe et al. 2020). O estudo também mostrou que a qualificação acadêmica das enfermeiras foi um fator que favoreceu a adesão às boas práticas de higienização das mãos.

Os achados desta revisão bibliográfica corroboram com estudos anteriores sobre o conhecimento e adesão dos profissionais de saúde à higienização das mãos (Efstathiou et al. 2011; Fayaz et al. 2014; Salmam et al. 2018). Dos dez estudos relados nesta revisão avaliando diferentes grupos de profissionais, seis mostraram que os médicos apresentam frequência significantemente alta de higienização das mãos, enquanto apenas três estudos revelaram que os enfermeiros estão à frente nesta prática. Esses dados são preocupantes, pois enfermeiros, técnicos e auxiliares lidam diretamente com pacientes, por isso a frequência de higienização das mãos por esses grupos de profissionais deve ser significantemente alta, semelhante ou até maior as observadas em médicos. A menor adesão observada nos enfermeiros pode estar relacionada a sobrecarga de trabalho gerada pelo grande número de pacientes hospitalizados (Afemikhe et al. 2020). Segundo Salmam et al. (2018) os profissionais de saúde relatam esquecimento e pressão durante o tempo de trabalho para justificar a pouca higienização das mãos. Neste sentido, proporcionar treinamentos para enfatizar a importância da higienização das mãos como barreira contra microrganismos (Subbalakshmi et al. 2020), assim como fixar cartazes nos departamentos dos hospitais como lembrete aos profissionais de saúde para que higienizem as mãos pode aumentar a adesão à prática (Salmam et al. 2018). Abbas et al. (2020) reforça que as unidades de saúde são responsáveis por realizar treinamentos rotineiros entre as equipes sobre as práticas de prevenção e controle de infecção, bem como favorecer um ambiente de trabalho com condições para realização das práticas, ressaltando a importância do abastecimento de sabonetes e degermantes nas pias em lugares estratégicos e álcool em gel.

\section{Momento da higienização das mãos}

Em 2020 a OMS lançou a campanha "lave as mãos, salve vidas" (OMS, 2020) a fim de reduzir a transmissão e contágio pelo novo coronavírus, no entanto, os resultados desta revisão mostram que a higienização das mãos pelos profissionais de saúde acontece, na grande parte das vezes, apenas mediante ao contato com o paciente (Agarwal et al. 2021; Husain 2020; Derksen, Keller \& Lippke, 2020; Al Abri, Al Zeedi \& Al Lawati, 2021; Afemikhe et al. 2020; Abbas et al. 2020; Huang, Q et al. 2021).

Agarwal et al. (2021) mostraram que nos meses de julho e agosto de 2020, frequentemente, 78,56\% dos profissionais de saúde de diferentes lugares da Índia cumprimentavam os colegas com aperto de mãos, 62,76\% tocavam nariz, boca e olhos sem higienizar as mãos, apenas 52,82\% seguiam as normas de higienização das mãos preconizadas pela OMS e 67,89\% higienizavam as mãos após ter contato com pacientes. Considerando que o SARS-CoV-2 é transmitido por gotículas e contado direto, a menor frequência da higienização das mãos neste caso favorece o contágio não só entre profissionais como também de profissionais para pacientes e vice-versa. Hussain et al. (2020) avaliando somente médicos observaram que a adesão da higienização das mãos após o contato com pacientes era significativa, afirmada por $98 \%$ dos entrevistados. Segundo Ashinyo et al. (2021) 88.4\% realizavam a higienização das mãos seguindo a preconização da OMS. 97,3\% dos profissionais afirmaram higienizar as mãos após tocar em pacientes com COVID-19, 93,9\% antes ou após realizar procedimentos, 95,1\% após se expor a fluídos corporais e 97,3\% após tocar em superfície de grande contato. Observando 267 vezes os profissionais de saúde, visando identificar os momentos de higienização das mãos por câmeras de vigilância, Derksen, Keller \& Lippke (2020) 
confirmaram os achados de Ashinyo et al. (2021), uma vez que em 75 vezes (28\%) os profissionais lavaram as mãos antes do contato com pacientes e 95 (34\%) vezes após encerrar o contato.

Avaliando a qualidade da higienização das mãos de 119 profissionais de saúde, Maude et al. (2021) observaram que apenas 57,9\% higienizavam todas as áreas das mãos. Sem especificar o momento que profissionais de saúde da Arábia Saudita higienizavam as mãos, Shaikhain et al (2021) mostrou que 70\% tinham boa adesão, no entanto, apenas 63\% sabiam o tempo correto de higienização. A OMS recomenda que a lavagem das mãos com sabonete seja de 40-60 segundos e a higienização com álcool em gel seja de 20-30 segundos (OMS, 2020). Esse tempo deve ser respeitado para que todas as partes das mãos sejam limpas e fique livre de microrganismos (Mertz et al. 2010). Maude et al. (2021) mostrou, ainda, que a região dorsal entre os dedos é a menos higienizada, isso facilita a contaminação pelo SARS-CoV-2 quando o profissional teve contato com o vírus. Já Tan et al. (2020a) avaliando 673 profissionais de um hospital, observou que apenas 72,9\% higienizavam as mãos após tossir, coçar o nariz ou espirrar. 81,4\% dos entrevistados disseram lavar as mãos quando tocavam em superfícies contaminadas. Esse último resultado pode ser grave se salientarmos que não conseguimos controlar a assepsia de um ambiente sem ter conhecimento de quando foi limpo e manipulado, por tanto, todo ambiente deve ser encarado como contaminado até que se constate assepsia e dessa forma a higienização das mãos durante a pandemia da COVID-19 deve ser feita sempre que houver contato com uma superfície passível de contaminação.

Moreno-Casbas (2020) foi o único estudo que mostrou maior frequência de higienização das mãos após a exposição de fluídos (95,5\%) se comparado com antes e depois de tocar o paciente, $89,6 \%$ e 94,5\%, respectivamente. Os autores também mostraram que não houve diferenças para o uso de álcool em gel ou sabonete na higienização das mãos, confirmando os achados de Abbas et al. (2020), que observaram o emprego de ambos os agentes químicos em diferentes momentos da higienização das mãos. Já Ramadan et al. (2021) observou que a medida preventiva de médicos plantonistas mais praticada foi a higienização das mãos com água e desinfetante por mais de 20 segundos $(47,4 \%)$.

Os estudos de Huang, Q et al. (2021) e El-Sokkary et al. (2021) avaliaram a adesão à higienização das mãos entre profissionais que foram ou não infectados. El-Sokkary et al. (2021) entrevistou 77 profissionais que foram infectados e 308 não infectados e observou taxa de adesão menor entre os profissionais infectados (77,2\%) quando comprados aos não infectados (86,7\%). Huang, Q et al. (2021) mostraram uma associação direta da baixa adesão à higienização ao maior número de infectados. Já Al Abri, Al Zeedi \& Al Lawati (2021) evidenciaram ótima adesão pelos profissionais infectados (96,8\%). Os autores mostram também que as mulheres enfermeiras foram os profissionais infectados que apresentaram maior nível de ansiedade, o que foi responsável pela baixa adesão à higienização das mãos (Huang, Q et al. 2021). Estudos evidenciaram que a Síndrome de Burnout, causada pela sobrecarga de trabalho, está relacionada a menor adesão as práticas de controle de infecção pelos profissionais da linha de frente na pandemia (Tan et al. 2020b; Matsuo et al. 2020).

\section{Períodos da Pandemia}

Os poucos estudos presentes na literatura que avaliaram a adesão à higienização das mãos comparando os períodos antes e durante a pandemia, bem como as fases da pandemia, mostraram uma adesão mais significativa de higienização das mãos dentro dos hospitais no ano de 2020 (Huang, F et al. 2021; Moore et al. 2021; Arriba-Fernandez, Molina-Cabrillana \& Majem, 2021; Ochoa-Hein et al. 2020; Jin et al. 2020; Abbas et al. 2020; Derksen, Keller \& Lippke, 2020). Segundo Samraj et al. (2008), antes da pandemia a frequência de higienização das mãos pelos profissionais de saúde era menor que 50\%. Dessa forma, esse aumento está relacionado a muitos fatores, como por exemplo:campanhas durante a pandemia, oferecimento de cursos que abordam as técnicas de higienização das mãos e sua importância para controle da pandemia, o crescente número de infectados, o medo dos profissionais em serem contaminados e transmitir o vírus aos familiares e contactantes. 
Huang, F et al. (2021) realizaram um estudo por meio de um sistema que monitora o uso de álcool em gel na entrada e saída das enfermarias entre setembro de 2019 e novembro de 2020. A frequência média de uso de álcool ao entrar na enfermaria foi $23,88 \%$ antes da pandemia, $27,31 \%$ na primeira onda da pandemia e $13,7 \%$ na segunda onda. Ao sair da enfermaria a frequência de higienização mostrou-se maior, sendo 50,63\% antes da pandemia, $64,37 \%$ na primeira onda e $57,32 \%$ na segunda onda. Higienizar as mãos antes de entrar na enfermaria aumenta a proteção ao paciente e ao sair reduz o risco de contaminação do profissional de saúde (Huang, Q et al., 2021). Resultados semelhantes foram observados por Derksen, Keller \& Lippke (2020), que evidenciaram maior frequência de higienização das mãos no período de maior precaução para controle da pandemia. Harper et al. (2020) mostraram que o medo da pandemia encorajou os profissionais de saúde a seguirem as recomendações de saúde pública, mas que as emoções negativas reduziram a motivação. Isso explica o aumento das taxas de adesão à higienização das mãos na primeira onda da pandemia comparado ao período pré-Covid e a redução desta adesão comparado à segunda onda da pandemia. Moore et al. (2021) monitoraram o consumo de álcool em gel e sabonete disponíveis em 9 unidades hospitalares do Reino Unido entre janeiro e maio de 2020. Os períodos de análise foram divididos em dois, antes e após o fechamento das escolas, que ocorreu no período mais crítico da pandemia, dessa forma os autores observaram que a quantidade de álcool e sabonete nos frascos estava maior durante o período que as escolas estavam fechadas, enquanto a frequência de higienização das mãos aumentou de 48,52\% antes das escolas fecharem para 58,05\% após o fechamento. Segundo autores, durante o período de fechamento das escolas, as visitas a pacientes diminuíram, o que favoreceu a redução do uso de álcool em gel e sabonete na entrada e saída das enfermarias. Resultados que mostram o aumento da adesão à higienização das mãos também foram evidenciados por Arriba-Fernandez, Molina-Cabrillana \& Majem (2021) comparando os anos de 2018, 2019 e 2020, sendo o ano de 2020 o de maior adesão com uma taxa de 59,2\%, 2019 com 47,6\% e 2018 com taxa de adesão pouco significativa, 42,5\%. Já Ochoa-Hein et al. (2020) mostraram taxa de adesão mais elevada em 2020, 94\% após adequação das normas estabelecidas por órgãos públicos comparados com $66,1 \%$ antes da adequação, entre janeiro de 2019 e fevereiro de 2020. Profissionais avaliados antes e depois de receber treinamento sobre a importância do controle de infecção apresentaram maior frequência de higienização das mãos depois de receber o treinamento (Abbas et al., 2020).

Jin et al. (2020) não evidenciaram diferenças entre os resultados de antes e durante a pandemia do Sars-CoV-2, sendo a média de frequência de adesão à higienização das mãos $81,6 \%$ e $82,5 \%$, respectivamente. A OMS alerta que a pressão para executar práticas simples do dia a dia no controle de infecção como a higienização das mãos deixa os profissionais de saúde estressados, reduzindo a taxa de adesão durante a pandemia (OMS, 2020), além disso, autores também alertam para o cuidado da saúde física e mental dos profissionais de saúde que estão na linha de frente na pandemia como fatores importantes que favorecem as boas práticas de higienização das mãos (Ayanian, 2020).

\section{Conclusão}

A taxa de adesão à higienização das mãos na pandemia mostrou-se significantemente maior em relação ao período pré-COVID-19. Os médicos e enfermeiros são os profissionais que higienizam as mãos com maior frequência durante a pandemia, principalmente após o contato com pacientes. Já os técnicos e auxiliares de enfermagem mostraram-se menos adeptos, o que é preocupante, uma vez que esses profissionais estão diretamente envolvidos com o cuidado integral de pacientes.

A frequência de higienização foi maior nos picos de transmissão do SARS-CoV-2, nesse sentindo concluímos que é fundamental monitorar e promover educação continuada a todos os profissionais do âmbito hospitalar de forma a enfatizar a importância e a execução da prática no combate do novo coronavírus. 


\section{Referências}

Abbas, K., Nawaz, S., Amin, N., Soomro, F. M., Abid, K., Ahmed, M., Sayeed, K. A., Ghazanfar, S., \& Qureshi, N. (2020). A web-based health education module and its impact on the preventive practices of health-care workers during the COVID-19 pandemic. Health education research, 35(5), 353-361.

Afemikhe, J. A., Esewe, R. E., Enuku, C. A., \& Ehwarieme, T. A. (2020). Transmission Based Precaution Practices among Nurses in Edo State, Nigeria during COVID-19 Pandemic. African journal of reproductive health, 24(s1), 98-107.

Agarwal, A., Ranjan, P., Saraswat, A., Kasi, K., Bharadiya, V., Vikram, N., Singh, A., Upadhyay, A. D., Baitha, U., Klanidhi, K. B., \& Chakrawarty, A. (2021). Are health care workers following preventive practices in the COVID-19 pandemic properly? A cross-sectional survey from India. Diabetes \& metabolic syndrome, 15(1), 69-75.

Ahmed, J., Malik, F., Memon, Z. A., Bin Arif, T., Ali, A., Nasim, S., Ahmad, J., \& Khan, M. A. (2020). Compliance and Knowledge of Healthcare Workers Regarding Hand Hygiene and Use of Disinfectants: A Study Based in Karachi. Cureus, 12(2), e7036.

Aidos, H., Diogo, P., \& Santos, J. M. (2018). Root Resorption Classifications: A Narrative Review and a Clinical Aid Proposal for Routine Assessment. European endodontic journal, 3(3), 134-145.

Aiello, A. E., Coulborn, R. M., Perez, V., \& Larson, E. L. (2008). Effect of hand hygiene on infectious disease risk in the community setting: a metaanalysis. American journal of public health, 98(8), 1372-1381.

Al Abri, Z., Al Zeedi, M., \& Al Lawati, A. A. (2021). Risk Factors Associated with COVID-19 Infected Healthcare Workers in Muscat Governorate, Oman. Journal of primary care \& community health, 12, 2150132721995454.

Alba, S., Bakker, M. I., Hatta, M., Scheelbeek, P. F., Dwiyanti, R., Usman, R., Sultan, A. R., Sabir, M., Tandirogang, N., Amir, M., Yasir, Y., Pastoor, R., van Beers, S., \& Smits, H. L. (2016). Risk Factors of Typhoid Infection in the Indonesian Archipelago. PloS one, $11(6)$, e0155286.

Ashinyo, M. E., Dubik, S. D., Duti, V., Amegah, K. E., Ashinyo, A., Asare, B. A., Ackon, A. A., Akoriyea, S. K., \& Kuma-Aboagye, P. (2021). Infection prevention and control compliance among exposed healthcare workers in COVID-19 treatment centers in Ghana: A descriptive cross-sectional study. PloS one, 16(3), e0248282

Ayanian JZ. (2020). Mental Health Needs of Health Care Workers Providing Frontline COVID-19 Care. JAMA Health Forum, 1 (4), e200397.

Barnes, S. L., Morgan, D. J., Harris, A. D., Carling, P. C., \& Thom, K. A. (2014). Preventing the transmission of multidrug-resistant organisms: modeling the relative importance of hand hygiene and environmental cleaning interventions. Infection control and hospital epidemiology, 35(9), 1156-1162.

Boyce, J. M., Pittet, D., \& Healthcare Infection Control Practices Advisory Committee. Society for Healthcare Epidemiology of America. Association for Professionals in Infection Control. Infectious Diseases Society of America. Hand Hygiene Task Force (2002a). Guideline for Hand Hygiene in Health-Care Settings: recommendations of the Healthcare Infection Control Practices Advisory Committee and the HICPAC/SHEA/APIC/IDSA Hand Hygiene Task Force. Infection control and hospital epidemiology, 23(12 Suppl), S3-S40.

Boyce, J. M., Pittet, D., Healthcare Infection Control Practices Advisory Committee, \& HICPAC/SHEA/APIC/IDSA Hand Hygiene Task Force (2002b). Guideline for Hand Hygiene in Health-Care Settings. Recommendations of the Healthcare Infection Control Practices Advisory Committee and the HICPAC/SHEA/APIC/IDSA Hand Hygiene Task Force. Society for Healthcare Epidemiology of America/Association for Professionals in Infection Control/Infectious Diseases Society of America. MMWR. Recommendations and reports: Morbidity and mortality weekly report. Recommendations and reports, 51(RR-16), 1-CE4.

Chen, J. D., Yuan, J., He, Z., Yang, Z. C., \& Wang, M. (2011). Zhonghua yu fang yi xue za zhi [Chinese journal of preventive medicine], 45(2), 101-106.

de Arriba-Fernández, A., Molina-Cabrillana, M. J., \& Serra Majem, L. (2021). Evaluación de la adherencia a la higiene de manos en profesionales sanitarios en un hospital de tercer nivel en relación con la pandemia de SARS-CoV-2 [Evolution of adherence to hand hygiene in health care professionals in a third level hospital in relation to the SARS-CoV-2 pandemic]. Revista espanola de quimioterapia. publicacion oficial de la Sociedad Espanola de Quimioterapia, 34(3), 214-219.

Derksen, C., Keller, F. M., \& Lippke, S. (2020). Obstetric Healthcare Workers' Adherence to Hand Hygiene Recommendations during the COVID-19 Pandemic: Observations and Social-Cognitive Determinants. Applied psychology. Health and well-being, 12(4), 1286-1305.

Efstathiou, G., Papastavrou, E., Raftopoulos, V., \& Merkouris, A. (2011). Factors influencing nurses' compliance with Standard Precautions in order to avoid occupational exposure to microorganisms: A focus group study. BMC nursing, 10, 1.

Egbi, O. G., Duru, C., \& Kasia, B. (2020). Knowledge, attitude and practice towards COVID-19 among workers of a tertiary hospital in Bayelsa State, Nigeria. The Pan African medical journal, 37(Suppl 1), 24.

Ejemot-Nwadiaro, R. I., Ehiri, J. E., Arikpo, D., Meremikwu, M. M., \& Critchley, J. A. (2015). Hand washing promotion for preventing diarrhoea. The Cochrane database of systematic reviews, 2015(9), CD004265.

Elhadi, M., Msherghi, A., Alkeelani, M., Zorgani, A., Zaid, A., Alsuyihili, A., Buzreg, A., Ahmed, H., Elhadi, A., Khaled, A., Boughididah, T., Khel, S., Abdelkabir, M., Gaffaz, R., Bahroun, S., Alhashimi, A., Biala, M., Abulmida, S., Elharb, A., Abukhashem, M., \& Amshai, A. (2020). Assessment of Healthcare Workers' Levels of Preparedness and Awareness Regarding COVID-19 Infection in Low-Resource Settings. The American journal of tropical medicine and hygiene, 103(2), 828-833.

El-Sokkary, R. H., El-Kholy, A., Mohy Eldin, S., Khater, W. S., Gad, D. M., Bahgat, S., Negm, E., El Kholy, J. A., Mowafy, S., Mahmoud, E., \& Mortada, E. M. (2021). Characteristics and predicting factors of Corona Virus Disease-2019 (COVID-19) among healthcare providers in a developing country. PloS one, $16(1), \mathrm{e} 0245672$ 
Erasmus, V., Brouwer, W., van Beeck, E. F., Oenema, A., Daha, T. J., Richardus, J. H., Vos, M. C., \& Brug, J. (2009). A qualitative exploration of reasons for poor hand hygiene among hospital workers: lack of positive role models and of convincing evidence that hand hygiene prevents cross-infection. Infection control and hospital epidemiology, 30(5), 415-419.

Erasmus, V., Daha, T. J., Brug, H., Richardus, J. H., Behrendt, M. D., Vos, M. C., \& van Beeck, E. F. (2010). Systematic review of studies on compliance with hand hygiene guidelines in hospital care. Infection control and hospital epidemiology, 31(3), 283-294.

Fayaz, S. H., Higuchi, M., Hirosawa, T., Sarker, M. A., Djabbarova, Z., \& Hamajima, N. (2014). Knowledge and practice of universal precautions among health care workers in four national hospitals in Kabul, Afghanistan. Journal of infection in developing countries, 8(4), 535-542.

Felix, A. M. S., Victor, E. S., Malaguti, S. E. T., \& Gir, E. (2013). Individual, work-related and institutional factors associated with adherence to standard precautions. Journal of Infection Control, 2(2), 106-111.

Harper, C. A., Satchell, L. P., Fido, D., \& Latzman, R. D. (2020). Functional Fear Predicts Public Health Compliance in the COVID-19 Pandemic. International journal of mental health and addiction, 1-14. Advance online publication.

Huang, D. B., \& Zhou, J. (2007). Effect of intensive handwashing in the prevention of diarrhoeal illness among patients with AIDS: a randomized controlled study. Journal of medical microbiology, 56(Pt 5), 659-663.

Huang, Q., Luo, L. S., Wang, Y. Y., Jin, Y. H., \& Zeng, X. T. (2021). Gender Differences in Psychological and Behavioral Responses of Infected and Uninfected Health-Care Workers During the Early COVID-19 Outbreak. Frontiers in public health, 9, 638975.

Huang, F., Armando, M., Dufau, S., Florea, O., Brouqui, P., \& Boudjema, S. (2021). COVID-19 outbreak and healthcare worker behavioural change toward hand hygiene practices. The Journal of hospital infection, 111, 27-34.

Husain, S. A., Husain, S. A., Khan, O. U., D'Cruz, L., \& Allgar, V. (2021). Review of hygiene adaptations among UK doctors in controlling the spread of SARS-CoV-2 infection. Clinical medicine (London, England), 21(1), e20-e25.

Jarvis W. R. (2007). The Lowbury Lecture. The United States approach to strategies in the battle against healthcare-associated infections, 2006: transitioning from benchmarking to zero tolerance and clinician accountability. The Journal of hospital infection, 65 Suppl 2, 3-9.

Jin, Y. H., Huang, Q., Wang, Y. Y., Zeng, X. T., Luo, L. S., Pan, Z. Y., Yuan, Y. F., Chen, Z. M., Cheng, Z. S., Huang, X., Wang, N., Li, B. H., Zi, H., Zhao, M. J., Ma, L. L., Deng, T., Wang, Y., \& Wang, X. H. (2020). Perceived infection transmission routes, infection control practices, psychosocial changes, and management of COVID-19 infected healthcare workers in a tertiary acute care hospital in Wuhan: a cross-sectional survey. Military Medical Research, 7(1), 24.

Karakose, T., Malkoc, N. (2021). Behavioral and interpersonal effects of the covid-19 epidemic on frontline physicians working in emergency departments (EDs) and intensive care units (ICUs). Acta Medica Mediterranea, 37(1), 437-444.

Köche, J. C. (2016). Fundamentos de metodologia científica. Ed. Vozes. http://www.brunovivas.com/wp-content/uploads/sites/10/2018/07/K\%C3\% B6cheJos\%C 3\%A9-Carlos0D0AFundamentos-de-metodologia-cient\%C3\%ADfica-_teoria-da0D0Aci\%C3\%AAncia-e-inicia\%C3\%A7\%C3\%A3o-\%C3\%A 0 - pe squisa.pdf

Ludke, M. \& Andre, M. E. D. A. (2013). Pesquisa em educação: abordagens qualitativas. Ed. EPU.

Magill, S. S., Edwards, J. R., Bamberg, W., Beldavs, Z. G., Dumyati, G., Kainer, M. A., Lynfield, R., Maloney, M., McAllister-Hollod, L., Nadle, J., Ray, S. M., Thompson, D. L., Wilson, L. E., Fridkin, S. K., \& Emerging Infections Program Healthcare-Associated Infections and Antimicrobial Use Prevalence Survey Team (2014). Multistate point-prevalence survey of health care-associated infections. The New England journal of medicine, 370(13), 1198-1208.

Marra, A. R., Cal, R. G., Silva, C. V., Caserta, R. A., Paes, A. T., Moura, D. F., Jr, dos Santos, O. F., Edmond, M. B., \& Durão, M. S. (2009). Successful prevention of ventilator-associated pneumonia in an intensive care setting. American journal of infection control, 37(8), 619-625.

Marra A. R. (2016). Advances in infection control. Einstein (Sao Paulo, Brazil), 14(1), 108-109.

Matsuo, T., Kobayashi, D., Taki, F., Sakamoto, F., Uehara, Y., Mori, N., \& Fukui, T. (2020). Prevalence of Health Care Worker Burnout During the Coronavirus Disease 2019 (COVID-19) Pandemic in Japan. JAMA network open, 3(8), e2017271.

Maude, R. R., Jongdeepaisal, M., Skuntaniyom, S., Muntajit, T., Blacksell, S. D., Khuenpetch, W., Pan-Ngum, W., Taleangkaphan, K., Malathum, K., \& Maude, R. J. (2021). Improving knowledge, attitudes and practice to prevent COVID-19 transmission in healthcare workers and the public in Thailand. BMC public health, 21(1), 749 .

Melis, M., Di Giosia, M., \& Colloca, L. (2019). Ancillary factors in the treatment of orofacial pain: A topical narrative review. Journal of oral rehabilitation, 46(2), 200-207.

Mertz, D., Dafoe, N., Walter, S. D., Brazil, K., \& Loeb, M. (2010). Effect of a multifaceted intervention on adherence to hand hygiene among healthcare workers: a cluster-randomized trial. Infection control and hospital epidemiology, 31(11), 1170-1176.

Ministério da Saúde. (2019). Controle de infecção hospitalar: balanço e reflexões. https://www.gov.br/anvisa/pt-br/assuntos/noticias-anvisa/2019/controle-deinfeccao-hospitalar-balanco-e-reflexoes.

Moore, L. D., Robbins, G., Quinn, J., \& Arbogast, J. W. (2021). The impact of COVID-19 pandemic on hand hygiene performance in hospitals. American journal of infection control, 49(1), 30-33

Morawska, L., \& Cao, J. (2020). Airborne transmission of SARS-CoV-2: The world should face the reality. Environment international, 139, 105730. 
Moreno-Casbas, M. T., en nombre del Grupo SANICOVI y Grupo de profesionales de la salud trabajando en la pandemia COVID-19, \& Integrantes del Grupo SANICOVI (2020). Factors related to SARS-CoV-2 infection in healthcare professionals in Spain. The SANICOVI project. Factores relacionados con el contagio por SARS-CoV-2 en profesionales de la salud en España. Proyecto SANICOVI. Enfermeria clinica (English Edition), 30(6), 360-370.

Ochoa-Hein, E., Rajme-López, S., Rodríguez-Aldama, J. C., Huertas-Jiménez, M. A., Chávez-Ríos, A. R., de Paz-García, R., Haro-Osnaya, A., GonzálezColín, K. K., González-González, R., González-Lara, M. F., Ponce-de-León, A., \& Galindo-Fraga, A. (2021). Substantial reduction of healthcare facility-onset Clostridioides difficile infection (HO-CDI) rates after conversion of a hospital for exclusive treatment of COVID-19 patients. American journal of infection control, 49(7), 966-968.

Ortiz-Prado, E., Simbaña-Rivera, K., Gómez-Barreno, L., Rubio-Neira, M., Guaman, L. P., Kyriakidis, N. C., Muslin, C., Jaramillo, A., Barba-Ostria, C., Cevallos-Robalino, D., Sanches-SanMiguel, H., Unigarro, L., Zalakeviciute, R., Gadian, N., \& López-Cortés, A. (2020). Clinical, molecular, and epidemiological characterization of the SARS-CoV-2 virus and the Coronavirus Disease 2019 (COVID-19), a comprehensive literature review. Diagnostic microbiology and infectious disease, 98(1), 115094.

Pereira A. S. et al. (2018). Metodologia da pesquisa científica. UFSM. https://repositorio.ufsm.br/bitstream/handle/1/15824/Lic_Computacao_MetodologiaPesquisa-Cientifica.pdf?sequence $=1$

Pittet, D., Allegranzi, B., Sax, H., Dharan, S., Pessoa-Silva, C. L., Donaldson, L., Boyce, J. M., \& WHO Global Patient Safety Challenge, World Alliance for Patient Safety (2006). Evidence-based model for hand transmission during patient care and the role of improved practices. The Lancet. Infectious diseases, 6(10), 641-652.

Pittet, D., Mourouga, P., \& Perneger, T. V. (1999). Compliance with handwashing in a teaching hospital. Infection Control Program. Annals of internal medicine, 130(2), 126-130.

Pittet D. (2000). Improving compliance with hand hygiene in hospitals. Infection control and hospital epidemiology, 21(6), 381-386.

Prather, K. A., Wang, C. C., \& Schooley, R. T. (2020). Reducing transmission of SARS-CoV-2. Science (New York, N.Y.), 368(6498), 1422-1424

Ramadan, M., Hasan, Z., Saleh, T., Jaradat, M., Al-Hazaimeh, M., Bani Hani, O., Al-Tammemi, A. B., Shorman, E., Al-Mistarehi, A. H., \& Kheirallah, K. (2021). Beyond knowledge: Evaluating the practices and precautionary measures towards COVID-19 amongst medical doctors in Jordan. International journal of clinical practice, $75(6), \mathrm{e} 14122$.

Salman, M., Raza, M. H., Mustafa, Z. U., Shrestha, S., Ali, M., Faham, H., Asif, N., Shehzadi, N., \& Hussain, K. (2018). Knowledge, attitudes and practices of hand hygiene among Pakistani health professionals: A cross-sectional study. Journal of infection in developing countries, 12(1), 63-66.

Samraj, S., Westbury, J., Pallett, A., \& Rowen, D. (2008). Compliance with hand hygiene in a genitourinary medicine department. International journal of STD \& AIDS, 19(11), 782-783.

Seto, W. H., Tsang, D., Yung, R. W., Ching, T. Y., Ng, T. K., Ho, M., Ho, L. M., Peiris, J. S., \& Advisors of Expert SARS group of Hospital Authority (2003). Effectiveness of precautions against droplets and contact in prevention of nosocomial transmission of severe acute respiratory syndrome (SARS). Lancet (London, England), 361(9368), 1519-1520.

Shaikhain, T. A., Al-Husayni, F. A., Alhejaili, E. A., Al-Harbi, M. N., Bogari, A. A., Baghlaf, B. A., \& Alzahrani, M. S. (2021). COVID-19-Related Knowledge and Practices Among Health Care Workers in Saudi Arabia: Cross-sectional Questionnaire Study. JMIR formative research, 5(1), e21220.

Stella, S. A., Stace, R. J., Knepper, B. C., Reese, S. M., Keniston, A., Burden, M., \& Young, H. L. (2019). The effect of eye images and a social norms message on healthcare provider hand hygiene adherence. Infection control and hospital epidemiology, 40(7), 748-754.

Subbalakshmi, E., Abirami, P., Subramanian, V., Victor, H. K. (2020). Awareness of hand hygiene in hospital set-up for infection control: Knowledge-based questionnaire for health care workers in a teaching hospital. Biomedical and Pharmacology Journal, 13(3).

Tan, B., Kanneganti, A., Lim, L., Tan, M., Chua, Y. X., Tan, L., Sia, C. H., Denning, M., Goh, E. T., Purkayastha, S., Kinross, J., Sim, K., Chan, Y. H., \& Ooi, S. (2020b). Burnout and Associated Factors Among Health Care Workers in Singapore During the COVID-19 Pandemic. Journal of the American Medical Directors Association, 21(12), 1751-1758.e5.

Tan, W., Hao, F., McIntyre, R. S., Jiang, L., Jiang, X., Zhang, L., Zhao, X., Zou, Y., Hu, Y., Luo, X., Zhang, Z., Lai, A., Ho, R., Tran, B., Ho, C., \& Tam, W. (2020). Is returning to work during the COVID-19 pandemic stressful? A study on immediate mental health status and psychoneuroimmunity prevention measures of Chinese workforce. Brain, behavior, and immunity, 87, 84-92.

Wang, X., Pan, Z., \& Cheng, Z. (2020). Association between 2019-nCoV transmission and N95 respirator use. The Journal of hospital infection, 105(1), 104105.

World Health Organization - WHO. Coronavirus disease (COVID-19) outbreak: rights,roles and responsibilities of health workers, including key considerations for occupational safety and health. 2020. https://www.who.int/publicationsdetail/coronavirus-disease-(covid-19)-outbreak-rights-rolesandresponsibilities-of-health-workers-including-key-considerations-foroccupational-safety-and-health.

Xun, Y., Shi, Q., Yang, N., Yang, N., Li, Y., Si, W., Shi, Q., Wang, Z., Liu, X., Yu, X., Zhou, Q., Yang, M., \& Chen, Y. (2021). Associations of hand washing frequency with the incidence of illness: a systematic review and meta-analysis. Annals of translational medicine, 9(5), 395. 